\title{
AVALIAÇÃO DE GENÓTIPOS DE BANANEIRA EM BOTUCATU-SP ${ }^{1}$
}

\author{
DAYANA PORTES RAMOS ${ }^{2}$, SARITA LEONEL ${ }^{3}$, \\ MARTHA MARIA MISCHAN ${ }^{4}$,ERVAL RAFAEL DAMATTO JÚNIOR ${ }^{5}$
}

RESUMO - O objetivo do trabalho foi avaliar o crescimento e a produção de genótipos de 3 grupos genômicos de bananeira: 'Nanicão-IAC-2001', 'Grande Naine', 'Caipira’e 'Nam'(AAA); 'Maçã', 'Thap Maeo', 'Prata-Anã' e 'Prata-Zulu' (AAB); 'FHIA 01', 'FHIA 18', 'Prata-Graúda' e 'Maçã Tropical' (AAAB), em Botucatu-SP. Foram avaliadas as seguintes características de crescimento: altura de plantas, circunferência do pseudocaule, número de folhas, número de dias entre o plantio e o florescimento, entre o florescimento e a colheita e do plantio à colheita. As características de produção avaliadas foram: peso do cacho, número de pencas, número de frutos, peso médio dos frutos, produtividade; peso, número, comprimento e diâmetro de frutos da $2^{\mathrm{a}}$ penca. Os resultados mostraram que, no grupo AAA, 'Nanicão' apresentou boas características de crescimento e produção, no entanto 'Grande Naine' apresentou maiores valores produtivos. 'Nam' apresentou menor porte e ciclo, porém baixos valores produtivos. No grupo AAB, 'Prata-Zulu' apresentou maior circunferência do pseudocaule, boas características produtivas, porém ciclo longo e porte maior. 'Thap Maeo' destacou-se por apresentar boas características de produção. 'Prata-Anã' apresentou porte reduzido, menor ciclo, porém não apresentou bom desempenho produtivo. 'FHIA 01', no grupo AAAB, apresentou maiores valores na maioria das características avaliadas. Pode concluir-se que 'Grande Naine' se destacou no grupo AAA, 'Thap Maeo' no grupo AAB e 'FHIA 01' no grupo AAAB.

Termos para indexação: Musa sp., crescimento, ciclo, produção.

\section{EVALUATION OF BANANA GENOTYPES IN BOTUCATU, STATE OF SÃO PAULO, BRAZIL}

\begin{abstract}
The purpose of this research was to assess the plants growth and production of genotypes of 4 genomic groups of banana: 'Nanicão-IAC-2001', 'Grande Naine', 'Caipira' and 'Nam' (AAA); 'Maçã', 'Thap Maeo', 'Prata Anã' and 'Prata Zulu'(AAB); 'FHIA 01', 'FHIA 18', 'Prata Graúda' and 'Maçã Tropical' (AAAB), in Botucatu, state of São Paulo, Brazil. Some growth characteristics were evaluated such as: plant height, the pseudostem circumference, number of leaves, number of days between planting and flowering, number of days between flowering and harvest and number of days from planting to harvest. The production characteristics such as the bunch weight, number of fruits, average weight of the fruits, number of hands and weight, number, length and diameter of the fruits of the $2^{\text {nd }}$ hand and yield. The results indicated that in the group AAA, 'Nanicão' showed good characteristics regarding to growth and production. However, 'Grande Naine' had the highest values in productive terms. 'Nam' showed the lowest height and the shortest cycle, but it had low productive values. Regarding to the group AAB, 'Prata Zulu' showed the biggest pseudostem circumference, good productive characteristics, but it had the longest cycle and the highest height. 'Thap Maeo' stands out as it shows good production characteristics. 'Prata Anã' had the lowest height and the shortest cycle. However, it did not have a good productive performance. In relation to the group AAAB, 'FHIA 01 ' showed the highest values in most of the characteristics which were evaluated, standing out among the other hybrids. We can conclude that 'Grande Naine' stands out inside the group AAA, 'Thap Maeo' and 'Prata Zulu' inside the group AAB and 'FHIA 01' inside the group AAAB.
\end{abstract}

Index terms: Musa sp., growth, cycle, yield.

\footnotetext{
1(Trabalho 261-08). Recebido em: 16-10-2008. Aceito para publicação em: 27-10-2009.

${ }^{2}$ Doutoranda pelo Departamento de Produção Vegetal-Horticultura, Bolsista CNPq, Faculdade de Ciências Agronômicas/FCA/UNESP - Cx. P. 237 - CEP 18610-307 - Botucatu-SP - pitchagro@yahoo.com.br.

${ }^{3}$ Prof a do Depto. de Produção Vegetal-Horticultura, FCA/UNESP - Cx. P. 237 - CEP 18610-307 - Botucatu-SP - sarinel@fca.unesp.br. ${ }^{4}$ Prof ${ }^{a}$ do Depto. de Bioestatística, Cx. P. 510 - CEP 18618-000 - Botucatu-SP - mmischan@ibb.unesp.br.

${ }^{5}$ Pesquisador do APTA, Rod. Régis Bittencourt, km 460, Cx. P. 122 - CEP 11900-000 - Registro-SP - erval@apta.sp.gov.br
} 


\section{INTRODUÇÃO}

A bananicultura brasileira possui grande importância econômica e social, sendo que o Brasil vem destacando-se no cenário mundial como o quarto maior produtor da fruta em 2007, segundo dados da FAO (2009), com produção em torno de 7,0 milhões de toneladas em uma área cultivada de 513 mil ha, sendo que o Estado de São Paulo se encontra entre os maiores produtores (Agrianual, 2008).

Apesar do grande número de cultivares existentes no Brasil, são poucos os que apresentam potencial agronômico para exploração comercial com alta produtividade, tolerância às pragas e doenças, porte reduzido e menor ciclo de produção.

Uma das estratégias para a solução dos problemas mencionados é a seleção de novas cultivares, mediante o melhoramento genético e, posteriormente, avaliação dessas cultivares em áreas de produção. Os caracteres normalmente estudados em avaliação de genótipos são o ciclo da cultura, altura da planta, perímetro do pseudocaule, peso do cacho, número de frutos por cacho, comprimento e diâmetro dos frutos, descritores relevantes para a identificação e a seleção de indivíduos superiores (Silva et al., 2000).

Sabendo do enorme potencial da bananicultura para o Estado de São Paulo e considerando-se a eminente expansão da cultura para áreas do planalto paulista, além do grande número de cultivares existentes no Brasil, somado à ausência de dados sobre essas cultivares em áreas de produção, o trabalho teve como objetivo avaliar o crescimento de plantas, ciclo vital e produção de genótipos de 3 grupos genômicos de bananeira: 'Nanicão-IAC-2001', 'Grande Naine', 'Caipira' e 'Nam' (AAA); 'Maçã', 'Thap Maeo', 'Prata-Anã' e 'Prata-Zulu' (AAB); 'FHIA 01', 'FHIA 18', 'Prata-Graúda' e 'Maçã Tropical' (AAAB), em Botucatu-SP.

\section{MATERIAL E MÉTODOS}

O experimento foi conduzido no período de janeiro de 2006 a janeiro de 2008, no pomar experimental do Departamento de Produção Vegetal - Horticultura, da Faculdade de Ciências Agronômicas - UNESP, Câmpus de Botucatu-SP, localizado nas coordenadas geográficas de $22^{\circ} 55^{\prime} 55^{\prime}$ ' latitude $\mathrm{S}, 48^{\circ} 26^{\prime} 22^{\prime}$ " longitude $\mathrm{W}$ e altitude de $810 \mathrm{~m}$. O tipo climático predominante no local é o temperado quente (mesotérmico) com chuvas no verão e seca no inverno (Cwa - Köeppen), tendo temperatura média anual de $20,5^{\circ} \mathrm{C}$ e precipitação pluviométrica média anual de $1.533 \mathrm{~mm}$ (Cunha et al., 1999). O solo da área foi classificado como Nitossolo Vermelho, se- gundo os critérios da Embrapa (1999).

As mudas, oriundas do processo de micropropagação, foram transplantadas para o campo, em área previamente preparada, num espaçamento de $2,5 \mathrm{x}$ $2,5 \mathrm{~m}$, com uma área de $6,25 \mathrm{~m}^{2} /$ planta. Durante o primeiro ciclo de produção, as plantas receberam os seguintes tratos culturais: irrigação complementar, controle de plantas daninhas, desbaste, retirada de folhas secas, eliminação do coração e corte do pseudocaule após a colheita.

Foi adotado o delineamento experimental inteiramente casualizado, com 12 tratamentos (genótipos-Tabela 1), 5 repetições e duas plantas úteis por parcela experimental, completamente rodeada por quatro plantas na bordadura. Foram realizadas as análises de variâncias e, quando houve significância, o teste de Tukey $(P \leq 0,05)$ foi utilizado para a comparação entre médias de genótipos, dentro de cada grupo genômico.

Foram avaliadas as seguintes características de crescimento: altura de plantas, circunferência do pseudocaule e número de folhas, medidas na época da emissão da inflorescência. Em relação ao ciclo da planta, avaliou-se o intervalo em dias entre o plantio e o florescimento, entre o florescimento e a colheita e entre o plantio e a colheita. Também foram mensuradas as seguintes características de produção: peso do cacho, número de frutos, peso médio dos frutos, número de pencas, peso, número, comprimento, diâmetro de frutos da $2^{\circ}$ penca e a produtividade.

\section{RESULTADOS E DISCUSSÃO}

Na Tabela 2, são apresentados os resultados referentes às características de crescimento da planta. Para altura da planta, observa-se que os genótipos que apresentaram o maior porte dentro de cada grupo genômico foram 'Grande Naine' (AAA), 'Prata-Zulu' (AAB) e 'Maçã Tropical' (AAAB). Os outros genótipos em estudo apresentaram porte variando de médio a baixo, sendo mais recomendado comercialmente, segundo Santos et al. (2006).

Leite et al. (2003), em Belmonte-BA, relataram que 'Grande Naine' apresentou uma altura parecida com a do presente trabalho $(2,6 \mathrm{~m})$. Fancelli (2003) confirma que a cultivar Prata-Zulu possui um porte alto. De acordo com Saes et al. (2005), 'Maçã Tropical' apresenta uma altura média de 3,2 m, corroborando os resultados deste trabalho.

Santos et al. (2006) salientam que, em uma cultivar comercial, é indesejável que a bananeira expresse valores muito elevados, pois pode dificultar a colheita e provocar o tombamento da planta em decorrência de ventos fortes e ataques de nematoides 
e broca. Além disso, a altura da planta é um descritor importante, tanto do ponto de vista fitotécnico como genético, permitindo maior adensamento e, consequentemente, maiores produtividades.

Para a circunferência do pseudocaule, no grupo $\mathrm{AAAB}$, os híbridos não diferiram entre si, notando que estes apresentaram valores maiores que os das cultivares triploides, o que permite inferências sobre a possível indicação de alguns híbridos para a diversificação de cultivares.

Silva \& Alves (1999) relatam que a circunferência do pseudocaule está relacionada ao vigor, e reflete a capacidade de sustentação do cacho, por isso genótipos que apresentam maior diâmetro são menos suscetíveis ao tombamento.

É interessante observar que 'Grande Naine' (AAA) e 'Prata-Zulu' (AAB) apresentaram maiores valores em relação à altura e vigor, indicando que apesar de apresentarem porte mais elevado, são capazes de sustentar seu cacho.

A média do número de folhas no florescimento é importante, pois reflete o potencial produtivo da variedade, que depende da taxa de fotossíntese e a tolerância às doenças, como, por exemplo, a Sigatoka-amarela (Alves, 1990). O maior número de folhas no florescimento sugere que o cacho poderá ter condições satisfatórias para o seu desenvolvimento, o que credencia esta característica como importante descritor na avaliação de genótipos (Silva et al., 2000). Neste trabalho, observa-se que as cultivares Grande Naine (grupo AAA) e os híbridos FHIA 01, FHIA 18 e Prata-Gráuda (grupo AAAB) apresentaram os melhores resultados.

Segundo Soto Ballestero (1992), a presença de mais de oito folhas no florescimento é um fator considerado como suficiente para o desenvolvimento normal do cacho, indicando que os genótipos avaliados apresentaram cachos bem desenvolvidos.

Na Tabela 3, são apresentados os resultados relacionados com o ciclo da planta e observa-se que houve diferenças significativas dentro de cada grupo genômico. A cultivar Nam apresentou menor período para florescer no grupo AAA, 'Prata-Anã' no grupo AAB, 'Maçã Tropical' e 'FHIA 01' no grupo AAAB.

Ledo et al. (1997) observaram, nas condições de Rio Branco-AC, que 'Nam' demorou 248 dias para florescer. No entanto, Pereira et al. (2003), em LavrasMG, relataram, para a mesma cultivar, 406 dias e para 'FHIA 01': 401 dias. Ganga et al. (2002), nas condições de Jaboticabal-SP, relataram 361 dias para 'FHIA 01' e Lima et al. (2005), em Cruz das AlmasBA, mostraram que 'Maçã Tropical' apresentou 276 dias, valor bem abaixo do presente trabalho.
Segundo Silva et al. (2000), o menor período para atingir o florescimento está relacionado com a precocidade do genótipo, sendo considerada uma característica importante, especialmente sob o ponto de vista econômico, pois resulta na obtenção de ciclos sucessivos de produção em menor espaço de tempo, aumentando a produção e a produtividade.

Os genótipos que apresentaram menores números de dias do florescimento à colheita foram: 'Nam' (AAA), 'Maçã' e 'Thap Maeo' (AAB), 'Maçã Tropical' e 'Prata-Graúda' (AAAB). Pereira et al. (2003), em Lavras-MG, relataram que 'Nam' apresentou 185 dias da floração à colheita. Gomes (2004), em Botucatu-SP, observou para 'Maçã': 101 dias. Andrade et al. (2002), em Teresina-PI, mostraram que 'Thap Maeo' apresentou 80 dias. Rodrigues et al. (2006), no norte de Minas Gerais, observaram para 'Prata-Graúda': 118 dias.

Segundo Damatto Júnior (2005), genótipos com menores intervalos entre o florescimento e a colheita apresentam a vantagem de menor permanência dos frutos em campo, ou seja, menores as chances de ocorrerem injúrias aos frutos, além de o retorno econômico ser mais rápido.

O ciclo apresenta uma fundamental importância no melhoramento genético da bananeira, pois é um caráter que expressa a precocidade. A redução do número de dias necessários para a emissão do cacho representa a antecipação do investimento aplicado (Santos et al., 2006). Notase que 'Nam' (AAA), 'Maçã' e 'Prata-Anã' (AAB), 'Maçã Tropical' (AAAB) destacaram-se por apresentar menor ciclo, sendo interessante para o produtor rural, pois este terá uma antecipação do seu investimento aplicado, bem como maior produção, em menor espaço de tempo.

Apesar de 'Prata-Anã' apresentar menor ciclo, foi a cultivar que apresentou maior número de dias entre o florescimento e a colheita, indicando que esses fatores não estão relacionados. Segundo Lima et al. (2005), os genótipos que apresentaram os maiores períodos do plantio à colheita, não foram os mesmos que apresentaram mais tempo para florescer, mostrando com isto que existem diferenças marcantes, entre os genótipos, no período que vai do florescimento à colheita.

Silva et al. (2000), em Cruz das Almas-BA, Leite et al. (2003), em Belmonte-BA e Pereira et al. (2003), em Lavras-MG, reportaram que 'Nam' apresentou um ciclo de 374; 466 e 590 dias, respectivamente. Segundo Damatto Júnior et al. (2005), em Botucatu-SP, Prata-Anã apresentou 496 dias do plantio à colheita. Pereira et al. (2003), em Lavras-MG e Rodrigues et al. (2006), no norte de Minas Gerais 
relataram para a mesma cultivar um ciclo de 510 e 320 dias, respectivamente. Leonel et al. (2004), em Botucatu-SP, observaram para a cv Prata-Anã, um ciclo de 434 dias e para o cv Maçã: 437 dias. Gomes (2004) relatou, para as mesmas condições e para as mesmas cultivares, menores valores, onde 'Maçã' apresentou um ciclo de 404 dias e 'Prata-Anã': 396 dias. Segundo Lima et al. (2005), em Cruz das Almas-BA e Silva et al. (2002b), em Petrolina-PE, 'Maçã Tropical' apresentou um ciclo de 376 e 384 dias, respectivamente.

Nota-se variação nos resultados referentes ao ciclo da planta, dependendo de cada região e até mesmo na mesma região, porém os maiores valores foram verificados em Botucatu-SP e Lavras-MG, devido apresentarem maiores altitudes e menores temperaturas, retardando o ciclo da planta, pois, segundo Gomes (1980), estes dois fatores exercem influência considerável sobre o fenótipo das plantas. É de concordância na literatura sobre a cultura que, a cada elevação de $100 \mathrm{~m}$ na altitude do local, onde a bananeira está sendo cultivada, ocorre proporcionalmente um incremento de 30 dias no ciclo vital da planta (Simão, 1998). Além do clima, adubações, tratamentos fitossanitátios, tratos culturais e diferenças clonais também podem interferir nas características avaliadas (Siqueira, 1984).

Na Tabela 4, são apresentados os resultados referentes a características de produção e observase que houve diferenças significativas para todos os parâmetros avaliados.

'Grande Naine' e 'Nanicão' (AAA), ‘PrataZulu' e 'Thap Maeo' (AAB), 'FHIA 01' e 'PrataGraúda' (AAAB) apresentaram maiores valores em relação ao peso dos frutos, do engaço, do cacho e consequentemente da produtividade. Percebe-se que esses genótipos mais produtivos apresentaram ciclos maiores, devido possuírem cachos mais pesados, consequentemente maior o tempo para a formação e enchimento dos frutos. Dentro desses genótipos, 'Grande Naine' (AAA), 'Thap Maeo' e 'FHIA 01' apresentaram um ciclo menor, sendo interessante para o produtor rural associar genótipos produtivos e precoces.

Segundo Saes et al. (2005), o peso do cacho de 'Nanicão-IAC-2001' variou entre 20,0 e 45,0 kg, corroborando os resultados deste trabalho. Carvalho et al. (2002) relataram que 'Grande Naine' apresentou produtividade de 37,4 t ha-1 e 'Nanicão': 34,5 t ha-1, em Teresina-PI. Leonel et al. (2004) e Barbosa (2008), em Botucatu-SP, reportaram para 'Nanicão' produtividades de 30,6 t ha- e 24,7 t ha' respectivamente.

Segundo Moreira (1999), 'Prata-Zulu' apresenta peso de cacho variando entre 20,0 e 25,0 kg. No entanto, Damatto Júnior et al. (2005), em Botucatu-SP, citaram que a bananeira 'Prata-Zulu' apresentou cachos pesando $33,9 \mathrm{~kg}$ e produtividade de $54,3 \mathrm{t} \mathrm{ha}^{-1}$. Leite et al. (2003), em Belmonte-BA, observaram que 'Thap Maeo' apresentou cachos pesando 16,0 kg. Segundo Carvalho et al. (2002), em Teresina-PI, a mesma cultivar apresentou produtividade de $19,5 \mathrm{t} \mathrm{ha}^{-1}$.

Observaram-se variações entre os resultados citados para os diferentes autores, até mesmo, na mesma região, e isto deve-se fundamentalmente aos fatores edáficos, climáticos, genéticos e ao manejo cultural adotado.

Pereira et al. (2003), em Lavras-MG, notaram que 'Prata-Graúda' apresentou cachos pesando 14,7 $\mathrm{kg}$ e 'FHIA 01': 12,3 kg; no entanto, Moura et al. (2002), em Itambé-PE, observaram para 'FHIA 01': $17,0 \mathrm{~kg}$ e para 'Prata-Graúda': 16,0 kg. Segundo Carvalho et al. (2002), em Teresina-PI, 'Prata-Graúda' apresentou produtividade média de $28,5 \mathrm{t} \mathrm{ha}^{-1}$. Notase que os valores citados no presente trabalho são superiores aos relatados por outros autores, indicando que os híbridos se adaptaram bem às condições de Botucatu-SP.

No grupo AAA, 'Grande Naine', 'Nanicão' e 'Caipira' apresentaram maior número de frutos/ cacho. No grupo AAB, 'Prata-Zulu' e 'Thap Maeo'. No grupo AAAB 'FHIA 01' e 'FHIA 18'.

Leonel et al. (2004), no muncípio de BotucatuSP, Lima et al. (2005), em Cruz das Almas-BA e Ganga et al. (2002), em Jaboticabal-SP, encontraram para a cultivar Nanicão 141; 136 e 116 frutos por cacho, respectivamente. Silva et al. (2004b) observaram, nas condições de Cruz das Almas-BA, que os valores variaram para 'Nanicão' de 165 a 110 frutos por cacho e para 'Grande Naine' de 163 a 95 frutos, mostrando que existem diferenças clonais nas cultivares.

Damatto Júnior et al. (2005), em Botucatu-SP, relataram que 'Prata-Zulu' apresentou 215 frutos/ cacho, mostrando valores superiores aos citados no presente trabalho. Ledo et al. (1997), em Rio BrancoAC, Leite et al. (2003), em Belmonte-BA e Silva et al. (2002a), em Cruz das Almas-BA, observaram que 'Thap Maeo' apresentou 168; 160 e 149 frutos/cacho, respectivamente.

Rodrigues et al. (2006), no norte de Minas Gerais, observaram que 'FHIA 01' apresentou 156 frutos e 'FHIA 18': 145. Pereira et al. (2003), em Lavras-MG, relataram que 'FHIA 01' mostrou-se com 96 frutos e 'FHIA 18': 92. Segundo Ganga et al. (2002), em Jaboticabal-SP, 'FHIA 01' apresentou 123 frutos e 'FHIA 18': 106. 
As citações variaram para cada região, sendo que Silva et al. (2000) relataram que outros estudos realizados no Brasil e no exterior constataram variações no número de frutos por cacho de distintos genótipos de bananeira, os quais foram atribuídos à sua constituição genética e às condições edafoclimáticas em que foram testados.

'Grande Naine' e 'Nanicão' (AAA), 'PrataGráuda' e 'FHIA 01' (AAAB) apresentaram as maiores médias de peso dos frutos. No grupo AAB, as cultivares não diferiram entre si.

Segundo Lima et al. (2005), 'Nanicão' apresentou frutos com peso médio de 128,2 g, em Cruz das Almas-BA. Donato et al. (2006), em Guanambi-BA, observaram que 'Grande Naine' apresentou frutos pesando 200,4 g e 'Nanicão': 194,0 g.

Rodrigues et al. (2006), no norte de Minas Gerais, relataram que o híbrido Prata-Graúda apresentou frutos pesando 213,0 g e os de 'FHIA 01': 182,0 g. Segundo Pereira et al. (2003), em Lavras-MG, os mesmos híbridos apresentaram frutos com peso de 157,1 e $127,2 \mathrm{~g}$, respectivamente.

Houve diferença nos resultados citados entre regiões e até na mesma região, cabendo as mesmas explicações dadas anteriormente.

'Grande Naine' e 'Nanicão' (AAA), 'PrataZulu' e 'Thap Maeo' (AAB), 'FHIA 01' e 'FHIA 18' foram os genótipos que apresentaram maior número de pencas.

Segundo Saes et al. (2005), 'NanicãoIAC-2001' produz até 11 pencas por cacho. Leonel et al. (2004), em Botucatu-SP, observaram que o cacho de 'Nanicão' apresentou 9 pencas; no entanto, Barbosa (2008) relatou 8 pencas. Para Gonzaga Neto et al. (1993), cachos de 'Grande Naine' e de 'Nanicão' apresentaram 9 pencas, no Submédio São Francisco.

Segundo Damatto Júnior et al. (2005), no município de Botucatu-SP, cachos de 'Prata-Zulu' apresentaram 14 pencas e Moreira (1999) relatou 10 pencas, corroborando os resultados deste trabalho. Segundo Silva et al. (2000), em Cruz das Almas-BA, e Ledo et al. (1997), em Rio Branco-AC, cachos de 'Thap Maeo' apresentaram 10 e 11 pencas, respectivamente.

Rodrigues et al. (2006) observaram, no norte de Minas Gerais, que 'FHIA 01' apresentou cachos com 11 pencas e 'FHIA 18': 10. Ganga et al. (2002), em Jaboticabal-SP, relataram para os mesmos híbridos 9 e 8 pencas, respectivamente.

As citações apresentadas estão próximas aos resultados encontrados neste trabalho.

No grupo AAA, 'Grande Naine' apresentou maior peso da $2^{a}$ penca, no grupo AAB, 'Thap Maeo' e 'Prata-Zulu', no grupo dos híbridos AAAB, 'PrataGraúda', 'FHIA 01', e 'Maçã Tropical'.

Gonzaga Neto et al. (1993), no Submédio São

Francisco, reportaram valores de penca para 'Grande Naine' próximas ao encontrado neste trabalho $(4,1$ $\mathrm{kg}$ ). Em condições de Botucatu-SP, Damatto Júnior et al. (2005) citaram para 'Prata-Zulu' peso de 2,9 kg. Ledo et al. (1997), em Rio Branco-AC, relataram que 'Thap Maeo' apresentou peso médio de pencas igual a 1,2 kg. Rodrigues et al. (2006), no norte de Minas Gerais, observaram que os híbridos Prata-Graúda e FHIA 01 apresentaram peso da $2^{\mathrm{a}}$ penca: $3,8 \mathrm{~kg}$.

No grupo AAB, 'Thap Maeo' apresentou maior número de frutos da $2^{\circ}$ penca. Os genótipos do grupo AAA e $\mathrm{AAAB}$ não diferiram entre si.

Leonel et al. (2004) e Barbosa (2008), em Botucatu-SP, observaram que os cachos de 'Nanicão' apresentaram 18 e 14 frutos na $2^{\mathrm{a}}$ penca, respectivamente. Gonzaga Neto et al. (1993), no Submédio São Francisco, reportaram que cachos de 'Grande Naine' e 'Nanicão' apresentaram 17 frutos/pcnca. Rodrigues et al. (2006), no norte de Minas Gerais, observaram 23 frutos na $2^{\text {a }}$ penca de 'Caipira', 19 na 'FHIA 01', 18 na 'FHIA 18' e 16 na 'Prata-Graúda', concordando com os dados deste trabalho.

'Grande Naine' apresentou frutos mais compridos, no grupo AAA. As cultivares do grupo AAB não diferiram entre si e apresentaram frutos medindo, em média, $15,5 \mathrm{~cm}$. No entanto, para o grupo AAAB, 'Prata-Graúda' e 'FHIA 01' apresentaram maiores valores.

Donato et al. (2006), em Guanambi-BA, e Carvalho et al. (2002), em Teresina-PI, relataram que 'Grande Naine' apresentou frutos com comprimento de $23,1 \mathrm{~cm}$ e $22,3 \mathrm{~cm}$, respectivamente, confirmando que esta cultivar possui frutos compridos.

Damatto Júnior et al. (2005), em BotucatuSP, citaram, para 'Prata-Zulu', comprimento de 14,3 cm e para 'Prata-Anã': 13,4 cm. Leonel et al. (2004) observaram frutos com comprimento de $12,6 \mathrm{~cm}$ para 'Prata-Anã' e 12,1 cm para 'Maçã', no município de Botucatu-SP. Segundo Gomes (2004), em Botucatu$\mathrm{SP}$, as mesmas cultivares mediram 12,0 e 10,7 cm, respectivamente. Segundo Leite et al. (2003), em Belmonte-BA, frutos de 'Maçã' apresentaram 16,0 cm, os de 'Prata-Anã': 14,0 cm e os de 'Thap Maeo': $14,7 \mathrm{~cm}$.

Rodrigues et al. (2006), no norte de Minas Gerais, observaram que 'Prata-Graúda' apresentou frutos com 16,8 cm de comprimento e 'FHIA 01': $16,1 \mathrm{~cm}$. Conforme Parrela et al. (2002), no norte de Minas Gerais, 'Prata-Graúda' apresentou frutos com 21,3 cm de comprimento e 'FHIA 01': 19,2 cm.

Novamente houve variação nos resultados 
para cada região e na mesma região, cabendo as mesmas explicações já expostas.

Os genótipos não diferiram entre si para nenhum grupo genômico, pois os frutos foram colhidos no estádio de desenvolvimento equivalente a “ $3 / 4$ gordo" (36-38 mm).

Segundo os resultados apresentados e discutidos, pode-se fazer um resumo para cada grupo genômico, com o intuito de verificar qual genótipo se destacou nas condições edafoclimáticas de Botucatu-SP.

No grupo genômico AAA, 'Nanicão' apresentou boas características de crescimento e de produção, porém apresentou ciclo longo. 'Grande Naine' apresentou boas características de crescimento e de produção. 'Caipira' somente se destacou em relação ao porte reduzido. 'Nam' apresentou menor porte e menor ciclo, no entanto apresentou baixos valores produtivos.

Para o grupo AAB, 'Maçã' apresentou ciclo curto, porém baixos valores produtivos. 'Thap Maeo' apresentou boas características produtivas. 'PrataAnã' apresentou porte reduzido, menor ciclo, no entanto não apresentou bom desempenho produtivo. 'Prata-Zulu' apresentou maior circunferência do pseudocaule, boas características produtivas, porém ciclo longo e porte maior.

Em relação aos híbridos, 'FHIA 01'apresentou as melhores características de crescimento e produção. 'FHIA 18' apresentou ciclo mais longo. 'Prata-Graúda' apresentou boas características de crescimento e produção. 'Maçã Tropical' apresentou o ciclo mais curto, porém mostrou-se com o porte elevado e os menores valores produtivos.

TABELA 1- Descrição dos genótipos de bananeira avaliados. Botucatu-SP, 2006-2008.

\begin{tabular}{|c|c|c|}
\hline Genótipos & Grupo & Descrição \\
\hline Nanicão-IAC-2001 & AAA & $\begin{array}{l}{ }^{1} \text { Subgrupo Cavendish, selecionado pelo IAC. É resistente à sigatoka- } \\
\text { amarela e tolerante à sigatoka-negra, imune ao mal-do-panamá e suscetível } \\
\text { ao moko, baixa tolerância ao ataque da broca-do-rizoma e do nematoide } \\
\text { cavernícola. }\end{array}$ \\
\hline Grande Naine & AAA & $\begin{array}{l}{ }^{2} \text { Subgrupo Cavendish. É suscetível às sigatokas, aos ne- } \\
\text { matoides e à broca-do-rizoma e resistente ao mal-do-panamá }\end{array}$ \\
\hline Caipira & AAA & $\begin{array}{l}{ }^{3} \text { Oriundo da África Ocidental e introduzido no Brasil pela Embrapa. É } \\
\text { resistente às sigatokas, ao mal-do-panamá e à broca-do-rizoma, suscetível } \\
\text { ao moko e ao nematoide cavernícola }\end{array}$ \\
\hline Nam & AAA & $\begin{array}{l}{ }^{4} \text { Introduzido da Tailândia, é resistente à sigatoka-amarela e ao mal-do- } \\
\text { panamá }\end{array}$ \\
\hline Maçã & $\mathrm{AAB}$ & $\begin{array}{l}{ }^{4} \text { É medianamente suscetível à sigatoka-amarela, medianamente resistente } \\
\text { à broca-do-rizoma, altamente suscetível ao mal-do-panamá e ao moko e } \\
\text { resistente aos nematoides }\end{array}$ \\
\hline Thap Maeo & $\mathrm{AAB}$ & $\begin{array}{l}{ }^{3} \text { Introduzido da Tailândia e selecionado pela Embrapa. Apresenta resis- } \\
\text { tência às sigatokas e ao mal-do-panamá, baixa incidência de broca-do- } \\
\text { rizoma e de nematoides }\end{array}$ \\
\hline Prata Anã & AAB & $\begin{array}{l}{ }^{4} \text { É suscetível às sigatokas, ao moko e ao mal-do-panamá, medianamente } \\
\text { resistente à broca-do-rizoma e resistente aos nematoides }\end{array}$ \\
\hline Prata Zulu & $\mathrm{AAB}$ & $\begin{array}{l}{ }^{3} \text { Originário da África. É altamente resistente às sigatokas, suscetível ao } \\
\text { moko, mal-do-panamá, broca-do-rizoma e ao nematoide cavernícola }\end{array}$ \\
\hline FHIA 01 & AAAB & $\begin{array}{l}{ }^{3} \text { Híbrido de Prata-Anã, introduzido de Honduras. É resistente às sigatokas } \\
\text { e suscetível ao moko }\end{array}$ \\
\hline FHIA 18 & AAAB & $\begin{array}{l}{ }^{4} \text { Híbrido de Prata-Anã, introduzido de Honduras. É resistente à sigatoka- } \\
\text { negra, medianamente resistente aos nematoides, medianamente suscetível } \\
\text { à sigatoka-amarela e à broca-do-rizoma, e suscetível ao moko e ao mal- } \\
\text { do-panamá }\end{array}$ \\
\hline Prata Graúda & AAAB & $\begin{array}{l}{ }^{4} \text { Híbrido de Prata-Anã, introduzido de Honduras. É resistente ao mal- } \\
\text { do-panamá, mediamanente suscetível à sigatoka- amarela e sucetível ao } \\
\text { moko e nematoides }\end{array}$ \\
\hline Maçã Tropical & AAAB & $\begin{array}{l}{ }^{4} \text { Híbrido criado pela Embrapa, semelhante à 'Maçã'. É resistente à } \\
\text { sigatoka-amarela, medianamente resistente aos nematoides, tolerante ao } \\
\text { mal-do-panamá e suscetível ao moko. }\end{array}$ \\
\hline
\end{tabular}

Fontes: ${ }^{1}$ Saes et al. (2005), ${ }^{2}$ Cordeiro (2003), ${ }^{3}$ Fancelli (2003) e ${ }^{4}$ Silva et al. (2004a). 
TABELA 2- Valores médios de altura de plantas, circunferência do pseudocaule e número de folhas por planta de genótipos de bananeiras de diferentes grupos genômicos, em Botucatu-SP (jan. 2006 a jan 2008).

\begin{tabular}{|c|c|c|c|}
\hline Genótipos & $\begin{array}{c}\text { Altura de plantas } \\
(\mathbf{m})\end{array}$ & $\begin{array}{l}\text { Circunferência do pseudocaule } \\
(\mathrm{cm})\end{array}$ & $\begin{array}{c}\text { Número de folhas por } \\
\text { planta }\end{array}$ \\
\hline \multicolumn{4}{|c|}{ GRUPO AAA } \\
\hline Nanicão & $2,4 \mathrm{AB}$ & $71,2 \mathrm{~B}$ & $11,8 \mathrm{AB}$ \\
\hline Grande Naine & $2,7 \mathrm{~A}$ & $76,7 \mathrm{~A}$ & $13,4 \mathrm{~A}$ \\
\hline Caipira & $2,2 \mathrm{~B}$ & $61,8 \mathrm{C}$ & $11,3 \mathrm{~B}$ \\
\hline Nam & $2,3 \mathrm{~B}$ & $63,2 \mathrm{C}$ & $10,5 \mathrm{~B}$ \\
\hline $\mathrm{CV}(\%)$ & 6,66 & 3,23 & 7,97 \\
\hline \multicolumn{4}{|c|}{ GRUPO AAB } \\
\hline Maçã & $2,5 \mathrm{~B}$ & $65,2 \mathrm{~B}$ & $11,8 \mathrm{~A}$ \\
\hline Thap Maeo & $2,6 \mathrm{~B}$ & $66,4 \mathrm{~B}$ & $11,2 \mathrm{~A}$ \\
\hline Prata-Anã & $2,1 \mathrm{C}$ & $67,7 \mathrm{~B}$ & $12,8 \mathrm{~A}$ \\
\hline Prata-Zulu & $3,0 \mathrm{~A}$ & $80,1 \mathrm{~A}$ & $12,4 \mathrm{~A}$ \\
\hline $\mathrm{CV}(\%)$ & 5,85 & 4,69 & 9,18 \\
\hline \multicolumn{4}{|c|}{ GRUPO AAAB } \\
\hline FHIA 01 & $2,6 \mathrm{~B}$ & $88,8 \mathrm{~A}$ & $11,8 \mathrm{~A}$ \\
\hline FHIA 18 & $2,8 \mathrm{~B}$ & $84,9 \mathrm{~A}$ & $11,8 \mathrm{~A}$ \\
\hline Prata-Graúda & $2,7 \mathrm{~B}$ & $82,5 \mathrm{~A}$ & $12,2 \mathrm{~A}$ \\
\hline Maçã Tropical & $3,2 \mathrm{~A}$ & $86,4 \mathrm{~A}$ & $10,0 \mathrm{~B}$ \\
\hline CV $(\%)$ & 3,61 & 4,27 & 7,04 \\
\hline
\end{tabular}

TABELA 3 - Valores médios de número de dias do plantio ao florescimento, número de dias entre o florescimento e a colheita, e número de dias do plantio à colheita (ciclo vital) de genótipos de bananeiras de diferentes grupos genômicos, em Botucatu-SP (jan. 2006 a jan. 2008).

\begin{tabular}{lccc}
\hline Genótipos & $\begin{array}{c}\mathbf{N}^{0} \text { de dias do plantio ao } \\
\text { florescimento }\end{array}$ & $\begin{array}{c}\mathbf{N}^{0} \text { de dias do florescimento } \\
\text { àcolheita }\end{array}$ & $\begin{array}{c}\mathbf{N}^{0} \text { de dias do plantio à } \\
\text { colheita (ciclo) }\end{array}$ \\
\hline GRUPO AAA & $448 \mathrm{~A}$ & $113 \mathrm{~A}$ & $561 \mathrm{~A}$ \\
\hline Nanicão & $429 \mathrm{AB}$ & $109 \mathrm{~A}$ & $538 \mathrm{~A}$ \\
Grande Naine & $416 \mathrm{AB}$ & $103 \mathrm{AB}$ & $519 \mathrm{AB}$ \\
Caipira & $398 \mathrm{~B}$ & $88 \mathrm{~B}$ & $486 \mathrm{~B}$ \\
Nam & 5,66 & 7,88 & 4,14 \\
\hline CV (\%) & & $90 \mathrm{C}$ & $462 \mathrm{~B}$ \\
\hline GRUPO AAB & $372 \mathrm{~B}$ & $109 \mathrm{C}$ & $522 \mathrm{~A}$ \\
\hline Maçã & $413 \mathrm{~A}$ & $126 \mathrm{~A}$ & $476 \mathrm{~B}$ \\
Thap Maeo & $350 \mathrm{C}$ & $118 \mathrm{~B}$ & $546 \mathrm{~A}$ \\
Prata-Anã & $428 \mathrm{~A}$ & 3,43 & 3,15 \\
Prata-Zulu & 4,37 & $135 \mathrm{~A}$ & $528 \mathrm{BC}$ \\
\hline CV (\%) & & $138 \mathrm{~A}$ & $573 \mathrm{~A}$ \\
\hline \multicolumn{5}{|c|}{ GRUPO AAAB } \\
\hline FHIA 01 & $393 \mathrm{~B}$ & $111 \mathrm{~B}$ & $531 \mathrm{~B}$ \\
FHIA 18 & $435 \mathrm{~A}$ & $103 \mathrm{~B}$ & $494 \mathrm{C}$ \\
Prata-Graúda & $420 \mathrm{~A}$ & 8,13 & 3,52 \\
Maçã-Tropical & $391 \mathrm{~B}$ & &
\end{tabular}


TABELA 4 - Valores médios de peso de frutos (PF), peso do engaço (PENG), peso do cacho (PC), número de frutos por cacho $\left(\mathrm{N}^{\circ} \mathrm{F}\right)$, peso médio do fruto $(\mathrm{PMF})$, produtividade $(\mathrm{PROD})$, número de pencas por cacho $\left(\mathrm{N}^{\mathrm{o}} \mathrm{P}\right)$, peso da $2^{\mathrm{a}}$ penca $\left(\mathrm{P} 2^{\circ} \mathrm{P}\right)$, número de frutos na $2^{\mathrm{a}}$ penca $\left(\mathrm{F} 2^{\circ} \mathrm{P}\right)$, comprimento (CF) e diâmetro de frutos (DF) de genótipos de bananeiras de diferentes grupos genômicos, em Botucatu-SP (jan. 2006 a jan. 2008).

\begin{tabular}{|c|c|c|c|c|c|c|c|c|c|c|c|}
\hline $\mathbf{G}$ & $\begin{array}{l}\mathrm{PF} \\
(\mathrm{kg})\end{array}$ & $\begin{array}{c}\text { PENG } \\
(\mathrm{kg})\end{array}$ & $\begin{array}{l}\mathrm{PC} \\
(\mathrm{kg})\end{array}$ & $\mathrm{N}^{\circ} \mathrm{F}$ & $\begin{array}{l}\text { PMF } \\
(\mathrm{g})\end{array}$ & $\begin{array}{l}\text { PROD } \\
\left(\mathrm{tha}^{-1}\right)\end{array}$ & $\mathrm{N}^{\mathrm{o}} \mathrm{P}$ & $\begin{array}{l}\mathrm{P} 2^{\circ} \mathrm{P} \\
(\mathrm{kg})\end{array}$ & $\mathrm{F} 2^{\circ} \mathrm{P}$ & $\begin{array}{c}\mathrm{CF} \\
(\mathrm{cm})\end{array}$ & $\begin{array}{c}\mathrm{DF} \\
(\mathrm{mm})\end{array}$ \\
\hline \multicolumn{12}{|c|}{ GRUPO AAA } \\
\hline 1 & $23,0 \mathrm{~A}$ & $2,1 \mathrm{AB}$ & $25,2 \mathrm{~A}$ & $163 \mathrm{~A}$ & $142,2 \mathrm{~A}$ & $36,9 \mathrm{~A}$ & & $2,9 \mathrm{~B}$ & 178 & $20,9 \mathrm{~B}$ & $36,0 \mathrm{~A}$ \\
\hline 2 & $29,3 \mathrm{~A}$ & $\mathrm{~A}$ & $31,9 \mathrm{~A}$ & ה & $152,8 \mathrm{~A}$ & $46,9 \mathrm{~A}$ & & $4,0 \mathrm{~A}$ & $22 \pi$ & $3 \mathrm{~A}$ & $5,5 \mathrm{~A}$ \\
\hline 3 & $13,3 \mathrm{~B}$ & $1,5 \mathrm{~B}$ & $14,9 \mathrm{~B}$ & $159 \mathrm{~A}$ & $83,8 \mathrm{~B}$ & $21,3 \mathrm{~B}$ & $8 \mathrm{BC}$ & $1,9 \mathrm{C}$ & $22 \mathrm{~A}$ & $14,7 \mathrm{C}$ & $32,0 \mathrm{~A}$ \\
\hline 4 & $8,4 \mathrm{~B}$ & $1,5 \mathrm{~B}$ & $9,9 \mathrm{~B}$ & 104B & $81,0 \mathrm{~B}$ & $13,5 \mathrm{~B}$ & $6 \mathrm{C}$ & $1,7 \mathrm{C}$ & $19 \mathrm{~A}$ & $13,8 \mathrm{C}$ & $34,0 \mathrm{~A}$ \\
\hline $\mathrm{CV}$ & 17,25 & 8,90 & 17,22 & 1,08 & 3,23 & 17,25 & 10,98 & 8,00 & 15,31 & 4,22 & 7,03 \\
\hline \multicolumn{12}{|c|}{ GRUPO AAB } \\
\hline 5 & $6,2 \mathrm{~B}$ & $0,8 \mathrm{C}$ & $7,0 \mathrm{~B}$ & $60 \mathrm{~B}$ & $104,1 \mathrm{~A}$ & $9,9 \mathrm{~B}$ & $6 \mathrm{~B}$ & $1,0 \mathrm{C}$ & 10D & $15,5 \mathrm{~A}$ & $35,6 \mathrm{~A}$ \\
\hline 6 & $16,1 \mathrm{~A}$ & $1,4 \mathrm{~B}$ & $17,6 \mathrm{~A}$ & $174 \mathrm{~A}$ & $92,3 \mathrm{~A}$ & $25,8 \mathrm{~A}$ & $12 \mathrm{~A}$ & $2,1 \mathrm{~A}$ & $20 \mathrm{~A}$ & $15,2 \mathrm{~A}$ & $35,8 \mathrm{~A}$ \\
\hline 7 & $8,4 \mathrm{~B}$ & $1,1 \mathrm{~B}$ & $9,5 \mathrm{~B}$ & $85 B$ & $99,3 \mathrm{~A}$ & $13,4 \mathrm{~B}$ & $7 \mathrm{~B}$ & $1,2 \mathrm{BC}$ & $13 \mathrm{C}$ & $16,0 \mathrm{~A}$ & $35,1 \mathrm{~A}$ \\
\hline 8 & $17,2 \mathrm{~A}$ & $1,9 \mathrm{~A}$ & $19,1 \mathrm{~A}$ & $80 \mathrm{~A}$ & $95,4 \mathrm{~A}$ & $27,5 \mathrm{~A}$ & $12 \mathrm{~A}$ & $1,6 \mathrm{AB}$ & $16 \mathrm{~B}$ & $15,0 \mathrm{~A}$ & $34,5 \mathrm{~A}$ \\
\hline CV & 19,59 & 8,54 & 19,08 & 14,34 & 2,45 & 19,59 & 10,63 & 9,31 & 11,53 & 5,74 & 3,18 \\
\hline \multicolumn{12}{|c|}{ GRUPO AAAB } \\
\hline 9 & $26,9 \mathrm{~A}$ & $3,3 \mathrm{~A}$ & $30,2 \mathrm{~A}$ & $167 \mathrm{~A}$ & $161,2 \mathrm{~A}$ & $43,1 \mathrm{~A}$ & $11 \mathrm{~A}$ & $3,3 \mathrm{~A}$ & $18 \mathrm{~A}$ & $22,4 \mathrm{~A}$ & $36,0 \mathrm{~A}$ \\
\hline 10 & $18,6 \mathrm{BC}$ & $2,1 \mathrm{~B}$ & $20,7 \mathrm{BC}$ & $163 \mathrm{~A}$ & $114,0 \mathrm{~B}$ & $29,8 \mathrm{BC}$ & $10 \mathrm{~A}$ & $2,1 \mathrm{~B}$ & $17 \mathrm{~A}$ & $18,5 \mathrm{~B}$ & $32,9 \mathrm{~A}$ \\
\hline 11 & $24,4 \mathrm{AB}$ & $2,5 \mathrm{~B}$ & $26,9 \mathrm{AB}$ & 137B & $179,7 \mathrm{~A}$ & $39,1 \mathrm{AB}$ & $9 \mathrm{~B}$ & $3,4 \mathrm{~A}$ & $17 \mathrm{~A}$ & $22,7 \mathrm{~A}$ & $37,3 \mathrm{~A}$ \\
\hline 12 & $16,7 \mathrm{C}$ & $1,9 \mathrm{~B}$ & $18,6 \mathrm{C}$ & 137B & $122,5 \mathrm{~B}$ & $26,7 \mathrm{C}$ & $8 \mathrm{C}$ & $2,8 \mathrm{~A}$ & $19 \mathrm{~A}$ & $17,8 \mathrm{~B}$ & $37,0 \mathrm{~A}$ \\
\hline $\mathrm{CV}$ & 14,92 & 7,71 & 14,42 & 9,38 & 3,23 & 14,92 & 6,45 & 6,77 & 7,82 & 6,15 & 7,83 \\
\hline
\end{tabular}

Medias seguidas pelas mesmas letras na coluna, para cada grupo genómico em separado, não diferem, pelo teste de lukey, a $5 \%$ de probabilidade. G: Genótipos. 1: Nanicão-IAC-2001. 2: Grande Naine. 3: Caipira. 4: Nam. 5: Maçã. 6: Thap Maeo. 7: Prata-Anã. 8: Prata-Zulu. 9: FHIA 01. 10: FHIA 18. 11: Prata-Graúda. 12: Maçã Tropical.

\section{CONCLUSÃO}

Os genótipos que se destacaram nos diferentes grupos genômicos foram: 'Grande Naine' (AAA), 'Thap Maeo' (AAB) e 'FHIA 01' (AAAB), pois dentro dos mais produtivos foram os que apresentaram menor ciclo.

\section{AGRADECIMENTOS}

À Fundação de Amparo à Pesquisa do Estado de São Paulo - FAPESP, pelo suporte financeiro que viabilizou a execução deste trabalho.

À Maria Izabel Furst Gonçalves, pela doação de parte do material vegetal.

\section{REFERÊNCIAS}

AGRIANUAL 2008: anuário estatístico da agricultura brasileira. São Paulo: FNP Consultoria \& Comércio, 2009. p. 196-203.

ALVES, E. J. Principais cultivares de banana no Brasil. Revista Brasileira de Fruticultura, Jaboticabal, v. 12, n. 3, p. 45-61, jan. 1990.

ANDRADE, G.M.; VASCONCELOS, M.E.C.; VELOSO, V.A.; SOUZA, B.; SOUSA, V.F. Avaliação de genótipos de bananeira no Estado do Piauí: comportamento vegetativo. In: CONGRESSO BRASILEIRO DE FRUTICULTURA, 17., 2002, Belém. Anais... Belém: SBF, 2002. CD-ROM.

BARBOSA, R. D. Manejo do solo com lodo de esgoto em bananeira irrigada. 2008. $84 \mathrm{f}$. Tese (Doutorado em Agronomia/Irrigação e Drenagem)Faculdade de Ciências Agronômicas, Universidade Estadual Paulista, Botucatu, 2008. 
CARVALHO, J.R.P., VASCONCELOS, L.F.L.; VELOSO, M.E.C.; SOUZA, V.A.B. ; CARVALHO, J.R.P. Avaliação de genótipos de bananeira no Estado do Piauí: comportamento produtivo. In: CONGRESSO BRASILEIRO DE FRUTICULTURA, 17., 2002, Belém. Anais... Belém: SBF, 2002. 1 CD-ROM.

CUNHA, A. R. et al. Classificação climática para o município de Botucatu-SP, segundo Köeppen. In: SIMPÓSIO EM ENERGIA NA AGRICULTURA, 1., 1999, Botucatu. Anais... Botucatu: Faculdade de Ciências Agronômicas, 1999. p. 490-491.

DAMATTO JÚNIOR, E. R. Efeitos da adubação com composto orgânico na fertilidade do solo, desenvolvimento, produção e qualidade de frutos de bananeira 'Prata-anã' (Musa AAB). 2005. 70 f. Dissertação (Mestrado em Agronomia/Energia na Agricultura) - Faculdade de Ciências Agronômicas, Universidade Estadual Paulista, Botucatu, 2005.

DAMATTO JÚNIOR, E. R. et al. Produção e caracterização de frutos de bananeira 'Prata-Anã' e 'Prata-Zulu'. Revista Brasileira de Fruticultura, Jaboticabal, v. 27, n. 3, p. 440-443, 2005.

DONATO, S. L. R.; SILVA, S. de O.; LUCCA FILHO, O.F.; LIMA, M.B.; DOMINGUES, H.; ALVES, J. da S. Comportamento de variedades e híbridos de bananeira (Musa spp.), em dois ciclos de produção no sudoeste da Bahia. Revista Brasileira de Fruticultura, Jaboticabal, v. 28, n. 1, p.139-144, 2006.

EMBRAPA. Centro Nacional de Pesquisa de Solos. Sistema brasileiro de classificação de solos. Rio de Janeiro, 1999. 412 p.

FANCELLI, M. Cultivo da bananeira para o Estado do Amazonas: cultivares. Cruz das Almas: EMBRAPA, CNPTIA, 2003. Disponível em: $<$ http://sistemasdeproducao.cnptia.embrapa.br/ FontesHTML/Banana/BananaAmazonas/ cultivares. htm>. Acesso em: 15 jul. 2007.

GANGA, R. M. D.; RUGGIERO, C.; MARTINS, A. B. G. Avaliação de seis cultivares de bananeira em Jaboticabal-SP. In: CONGRESSO BRASILEIRO DE FRUTICULTURA, 17., 2002, Belém. Anais... Belém: SBF, 2002. 1 CD-ROM.
GOMES, E. M. Crescimento e produção de bananeiras 'Prata-Anã'e 'Maçã' fertirrigadas com potássio. 2004. 76 f. Tese (Doutorado em Agronomia)Faculdade de Ciências Agronômicas, Universidade Estadual Paulista, Botucatu, 2004.

GOMES, W. R. Exigências climáticas da cultura da bananeira. Informe Agropecuário, Belo Horizonte, v. 6, n. 63 , p. $14-15,1980$.

GONZAGA NETO, L. et al. Avaliação de cultivares de bananeira na região do Submédio São Francisco: primeiro ciclo de produção. Revista Brasileira de Fruticultura, Jaboticabal, v. 15, n. 1, p. 21-25, 1993.

LEDO, A. da S.; SILVA, S. de O. e; AZEVEDO, F. F. de. Avaliação preliminar de genótipos de banana (Musa spp.) em Rio Branco-Acre. Revista Brasileira de Fruticultura, Jaboticabal, v. 19, n. 1, p. 51-56, 1997.

LEITE, J. B. V. et al. Caracteres da planta e do cacho de genótipos de bananeira, em 4 ciclos de produção, em Belmonte, Bahia. Revista Brasileira de Fruticultura, Jaboticabal, v. 25, n. 3, p. 443-447, 2003.

LEONEL, S.; GOMES, E. M.; PEDROSO, C. J. Desempenho agronômico de bananeiras micropropagadas em Botucatu-SP. Revista Brasileira de Fruticultura, Jaboticabal, v. 26, n. 2, p. 245-248, 2004.

LIMA, M. B. et al. Avaliação de cultivares e híbridos de bananeira no recôncavo baiano. Ciência e Agrotecnologia, Lavras, v. 29, n. 3, p. 515-520, 2005.

MOREIRA, R. S. Banana: teoria e prática de cultivo. 2. ed. São Paulo: Fundação Cargill, 1999. CD-ROM.

MOURA, R. J. M. et al. Avaliação de cultivares e híbridos de bananeira na zona da mata norte de Pernambuco ( $1^{\circ}$ ciclo). In: CONGRESSO BRASILEIRO DE FRUTICULTURA, 17., 2002, Belém. Anais... Belém: SBF, 2002. 1 CD-ROM.

PARRELA, R. A. da C. et al. Caracterização físicoquímica dos frutos de genótipos de bananeira produzidos no norte de Minas. In: CONGRESSO BRASILEIRO DE FRUTICULTURA, 17., 2002, Belém. Anais... Belém: SBF, 2002. 1 CD-ROM. 
PEREIRA, L. V. et al. Avaliação de cultivares e híbridos de bananeira em Lavras-MG. Ciência e Agrotecnologia, Lavras, v. 27, n. 1, p. 17-25, 2003.

RODRIGUES, M. G. V.; SOUTO, R. F.; SILVA, S. de O. e. Avaliação de genótipos de bananeira sob irrigação. Revista Brasileira de Fruticultura, Jaboticabal, v. 28, n. 3, p. 444-448, 2006.

SAES, L. A.; NOMURA, E. S.; GARCIA, V. A. Cultivares resistentes de bananeira. In: REUNIÃO ITINERANTE DE FITOSSANIDADE DO INSTITUTO BIOLÓGICO, 13., 2005, Registro. Anais... Registro: APTA, 2005. p. 51-58. Disponível em: $<$ http://www.biologico.sp.gov.br/rifib/XIIIRifib/saes. pdf $>$. Acesso em: 16 jul. 2007.

SANTOS, S.C.; CARNEIRO, L.C.; SILVEIRA NETO, A.M. da; PANIAGO JÚNIOR, E.; PEIXOTO, C.N. Caracterização morfológica e avaliação de cultivares de bananeira resistentes à Sigatoka-negra (Mycosphaerella fijiensis Morelet) no sudoeste goiano. Revista Brasileira de Fruticultura, Jaboticabal, v. 28, n. 3, p. 449-453, 2006.

SILVA, S. de O. e; ALVES, E. J. Melhoramento genético e novas cultivares de bananeira. Informe Agropecuário, Belo Horizonte, v. 20, n. 196, p. 91-96, 1999.

SILVA, S. de O. e; FLORES, J. C. de O.; LIMA NETO, F. P. Avaliação de cultivares e híbridos de bananeira em quatro ciclos de produção. Pesquisa Agropecuária Brasileira, Brasília, DF, v. 37, n. 11, p. 1567-1574, nov. 2002a.
SILVA, S. de O.; ALVES, E.J.; ANDRADE NETO, T.M. de; LICHTEMBERG, L.A.; FERREIORA, F.R. Avaliação de clones de bananeira do subgrupo Cavendish (Musa acuminata, AAA) em Cruz das Almas-BA. Ciência e Agrotecnologia, Lavras, v. 28, n .6, p. 1240-1246, nov./dez. 2004 .

SILVA, S. de O.; FLORI, J.E. ; PAIVA, L.E. ; ALVES, E.J. ; ANDRADE NETO, T. M. Avaliação de genótipos de bananeira tipo maçã em relação às características fitotécnicas e de reação ao maldo-panamá. In: CONGRESSO BRASILEIRO DE FRUTICUlTURA, 17., 2002b, Belém. Anais... Belém: SBF, 2002b. 1 CD-ROM.

SILVA, S. de O.; ROCHA, S.A.; ALVES, E. J.; CREDICO, M.; PASSOS, A. R. Caracterização morfológica e avaliação de cultivares e híbridos de bananeira. Revista Brasileira de Fruticultura, Jaboticabal, v. 22, n. 2, p. 161-169, 2000.

SIMÃO, S. Bananeira. In: Tratado de fruticultura. Piracicaba: FEALQ, 1998. p. 327 381.

SIQUEIRA, D. L. Variabilidade e correlação de caracteres em clones de bananeira 'Prata'. 1984. 66 f. Dissertação (Mestrado em Fitotecnia) - Escola Superior de Agricultura de Lavras, Lavras, 1984.

SOTO BALLESTERO, M. Bananas: cultivo y comercialización. 2. ed. San José: Litografia e Imprensa, 1992. $674 \mathrm{p}$. 